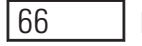

\section{ETHNIC DIFFERENCES IN CAROTID INTIMAL MEDIAL THICKNESS AND CAROTID-FEMORAL PULSE WAVE VELOCITY ARE PRESENT IN UK CHILDREN}

doi:10.1136/heartjnl-2011-300198.66

${ }^{1} \mathrm{P}$ H Whincup, ${ }^{1} \mathrm{C}$ M Nightingale, ${ }^{2} \mathrm{~A}$ Rapala, ${ }^{2} \mathrm{D}$ Joysurry, ${ }^{2} \mathrm{M}$ Prescott, ${ }^{2} \mathrm{~A} E$ Donald, ${ }^{2}$ E Ellins, ${ }^{1} \mathrm{~A}$ Donin, ${ }^{2} \mathrm{~S}$ Masi, ${ }^{1} \mathrm{C}$ G Owen, ${ }^{1} \mathrm{~A}$ R Rudnicka, ${ }^{1} \mathrm{D}$ G Cook, ${ }^{2} \mathrm{~J}$ E Deanfield. ${ }^{1}$ Division of Population Health Sciences, St George's, University of London, London, UK; ${ }^{2}$ Vascular Physiology Unit, Institute of Child Health, UCL, London, UK

Introduction There are marked ethnic differences in cardiovascular disease risks in UK adults; South Asians have high risks of coronary heart disease and stroke while black African-Caribbeans have high risks of stroke and slightly low risks of coronary heart disease when compared with white Europeans. Ethnic differences in cardiovascular risk factors are apparent in childhood, but little is known abut ethnic differences in vascular structure and function during childhood. We set out to measure two vascular markers of cardiovascular risk, common carotid intimal-medial thickness (cIMT) and carotidfemoral pulse wave velocity (PWV) in UK children from different ethnic groups.

Methods We conducted a school-based study examining the cardiovascular risk profiles of 9-10 year-old UK children, including similar numbers of South Asian, black African-Caribbean and white European participants. Following a baseline cardiovascular risk survey with measurements of body build, blood pressure, fasting blood lipids, insulin and $\mathrm{HbA1c}, 1400$ children were invited to have measurements of cIMT (bilateral measurements were made with a Zonare ultrasound scanner). A subgroup of these children $(n=900)$ was also invited for PWV measurements, made with a Vicorder device. All analyses were adjusted for age, gender and allowed for clustering at school level.

Results In all, 939 children (67\% response) had measurements of cIMT and 631 children (70\% response) had measurements of PWV. Mean cIMT was $0.475 \mathrm{~mm}$ (SD $0.035 \mathrm{~mm}$ ); mean PWV was $5.2 \mathrm{~m} / \mathrm{s}$ (SD $0.7 \mathrm{~m} / \mathrm{s}$ ). Compared with white European children, black African-Caribbeans had higher cIMT (mean difference $0.014 \mathrm{~mm}$, 95\%CI 0.008 to $0.021 \mathrm{~mm}$ ) and PWV (\% difference 3.3, 95\%CI 0.4 to 6.2); South Asian children had similar cIMT to white Europeans but slightly higher PWV (\% difference $2.7,95 \% \mathrm{CI}-0.1$ to $5.5 \%$ ). cIMT was positively associated with systolic and diastolic blood pressure but not with other cardiovascular risk markers. In contrast, PWV was positively associated with adiposity, diastolic blood pressure and insulin resistance. Black African-Caribbean children had lower LDL-cholesterol levels and higher insulin and HbA1c levels than white Europeans; South Asian children had higher insulin, HbA1c and triglyceride levels. However, adjustment for these risk factors had little effect on the ethnic differences in cIMT and PWV observed. Conclusions Ethnic differences in cIMT and PWV, markers of longterm cardiovascular risk, are apparent in childhood. These differences are not fully explained by the ethnic differences in established cardiovascular risk markers observed. The results suggest that there may be important opportunities for prevention of cardiovascular disease before adult life, particularly in high-risk ethnic minority groups.

\section{SPONTANEOUS CARDIAC HYPERTROPHY AND ADVERSE LV REMODELLING IN A NOVEL HUMAN RELEVANT MOUSE MODEL OF DIABETES; A MECHANISTIC INSIGHT}

doi:10.1136/heartjnl-2011-300198.67

${ }^{1} \mathrm{~S} M$ Gibbons, ${ }^{1} \mathrm{Z}$ Hegab, ${ }^{1} \mathrm{M} \mathrm{Zi},{ }^{1} \mathrm{~S}$ Prehar, ${ }^{1} \mathrm{~T} \mathrm{M}$ A Mohammed, ${ }^{2} \mathrm{R} \mathrm{D}$ Cox, ${ }^{1} \mathrm{E} J$ Cartwright, 'L Neyses, ${ }^{1} \mathrm{M}$ A Mamas. ${ }^{1}$ University of Manchester, Manchester, UK; ${ }^{2}$ MRC mammalian genetics unit, oxford, UK

Heart failure (HF) is one of the commonest cardiovascular complications of Diabetes Mellitus (DM) with the prevalence of DM reported at around 30\% in many pivotal heart failure studies. DM is an independent predictor of mortality in patients with HF, however molecular mechanisms that contribute to HF development in the diabetic population are poorly understood. Using a novel human relevant mouse model of DM (GENA348), identified through the MRC mouse mutagenesis programme with a point mutation in the pancreatic glucokinase (GLK) gene we investigate the molecular mechanisms that contribute to the HF phenotype in DM. GLK is the glucose sensor which regulates insulin secretion and GLK activity is reduced by $90 \%$ by the GENA348 point mutation resulting in severe hyperglycaemia. Similar mutations underlie Maturity Onset Diabetes of the Young Type 2 (MODY 2) in humans. Mean random blood glucose was found to be increased in the GENA348 mutant (HO) mice compared to wild type (WT) littermates (WT $6.9 \pm 0.3 \mathrm{mmol} / \mathrm{l}$ vs $\mathrm{HO} 20.6 \pm 0.8 \mathrm{mmol} / \mathrm{l}$, $\mathrm{p}<0.001$ ). Serial echocardiography was performed, at 3,6 and 12 months. No significant changes in echocardiographic parameters were observed at 3 months, although by 6 months development of significant cardiac hypertrophy in $\mathrm{HO}$ mice was observed. At 12 months of age left ventricular dilatation was evident, characterised by an $8 \%$ increase in diastolic diameter (WT $4.08 \pm 0.10$ vs $\mathrm{HO} 4.41 \pm 0.12, \mathrm{p}<0.05)$. Systolic function was preserved although significant diastolic dysfunction was evident at 6 and 12 months with a $31 \%$ reduction in the E:A ratio. Histological staining illustrated significant cellular hypertrophy with real time PCR data demonstrating a relative $150 \%$ increase in the hypertrophic marker BNP. Hypertrophic pathways were examined through western blot analysis revealing an age dependant increase in Akt phosphorylation (3 months- no increase, 6 months-140\%, 12 months-460\%). Serum levels of advanced glycation end products (AGE) were also elevated by $86 \%$ (WT $21 \pm 3.5 \mathrm{ng} / \mathrm{ml}$ vs $\mathrm{HO} 39 \pm 8.3 \mathrm{ng} / \mathrm{ml}, \mathrm{p}<0.05$ ) as was the protein expression level of the receptor for AGEs (RAGE). In vitro cellular experiments also revealed AGEs directly activate Akt through phosphorylation and increase levels of the receptor RAGE. AGE induced phosphorylation of Akt is inhibited in the presence of wortmannin, suggesting a PI3K dependent signalling mechanism. This was further confirmed in vivo where a bolus injection of wortmannin in 6-month old mutant mice returned Akt phosphorylation levels to those seen in WT mice. In conclusion, using the first human relevant mouse model of diabetes, GENA348 we demonstrate the development of a progressive cardiac phenotype including cardiac hypertrophy, LV dilatation and diastolic dysfunction similar to the clinical manifestations of diabetic cardiomyopathy. We propose that the RAGE/PI3K/Akt pathway contributes to the molecular mechanisms associated with the cardiac phenotype.

\section{RARE ALLELES IN GENETIC PREDISPOSITION TO CORONARY ARTERY DISEASE: INSIGHTS FROM THE NOVEL ANALYSIS OF GENE-CENTRIC ARRAY}

doi:10.1136/heartjnl-2011-300198.68

${ }^{1} \mathrm{P}$ Christofidou, ${ }^{1} \mathrm{R}$ Debiec, ${ }^{1} \mathrm{C}$ P Nelson, ${ }^{1} \mathrm{P}$ S Braund, , L D S Bloomer, ${ }^{2} \mathrm{~S}$ G Ball, ${ }^{2} \mathrm{~A} J$ Balmforth, ${ }^{2} \mathrm{~A} S$ Hall, ${ }^{1} \mathrm{M}$ Tomaszewski, ${ }^{1} \mathrm{~N} \mathrm{~J}$ Samani. ${ }^{1}$ University of Leicester, Leicester, UK; ${ }^{2}$ University of Leeds, Leeds, UK

Background Genome-wide association studies have been successful in identifying association between several common variants and coronary artery disease (CAD). However, collectively these variants explain only a small proportion of CAD heritability. It is becoming increasingly clear that the remainder of the "missing CAD heritability" could be explained by low frequency/rare alleles. Because of the small number of observations for any given rare allele, the power to detect its association with a phenotype is a major limiting factor in genetic analysis. In this study we have undertaken a novel statistical approach that combines information from all low frequency 